Supplement of Biogeosciences Discuss., 12, 19213-19267, 2015

http://www.biogeosciences-discuss.net/12/19213/2015/

doi:10.5194/bgd-12-19213-2015-supplement

(C) Author(s) 2015. CC Attribution 3.0 License.

(c) (1)

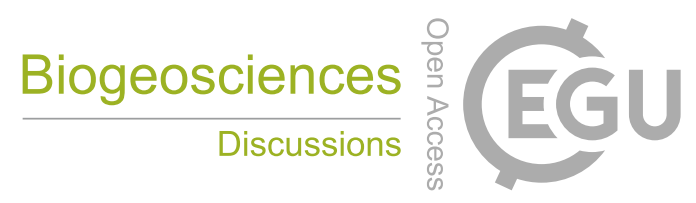

Supplement of

\title{
MODIS vegetation products as proxies of photosynthetic potential: a look across meteorological and biologic driven ecosystem productivity
}

\section{N. Restrepo-Coupe et al.}

Correspondence to: N. Restrepo-Coupe (nataliacoupe@gmail.com)

and A. Huete (alfredo.huete@uts.edu.au)

The copyright of individual parts of the supplement might differ from the CC-BY 3.0 licence. 
HSP: Tropical savanna 2000-2011

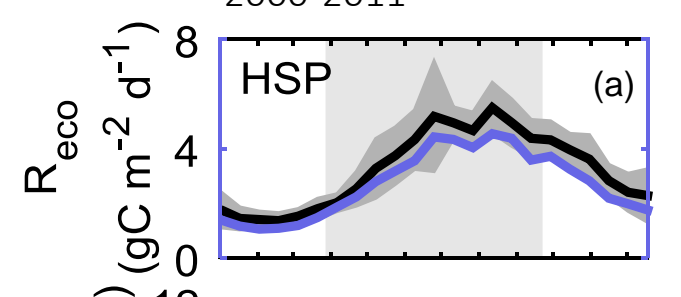

$\frown 12$

ס

แั้

ర్

o 0

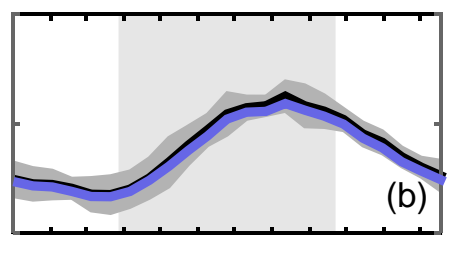

J JASOND J FMAM
TBR: Wet Sclerophyll

2000- 2010

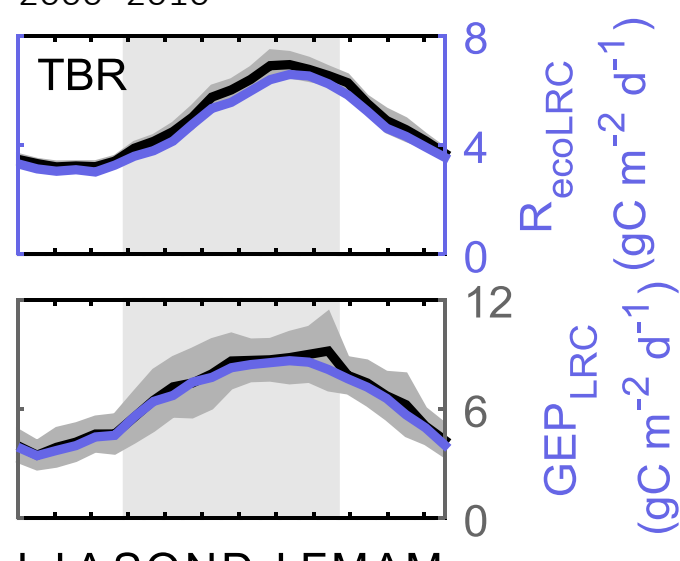

ASP: Mulga woodland 2010-2013

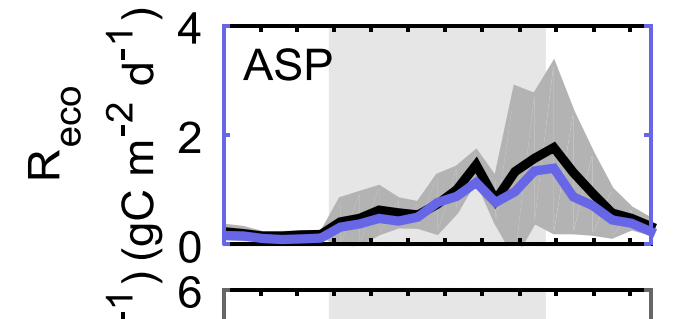

岗

ז

U్
CHO: Mediterranean - Mallee 2000-2013

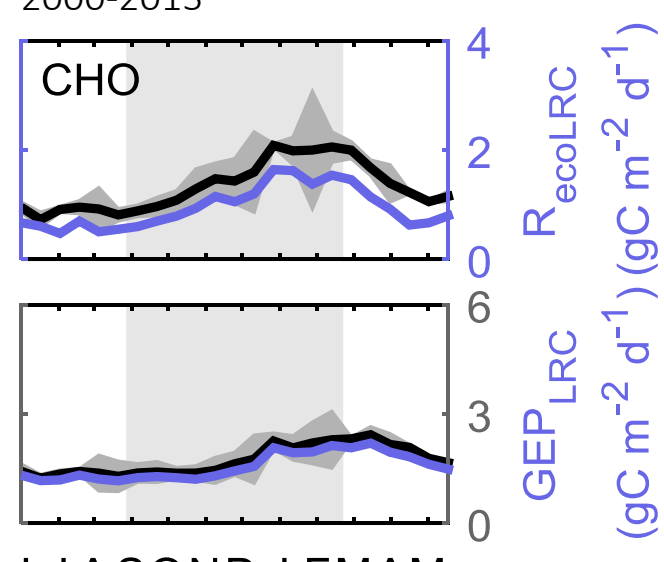

Supplement Figure 1. Savana, Mulga, Mallee and Wet Schlerophill ecosystems, Ozflux sites annual cycle (16-day composites) of (a) Ecosystem Respiration derived using a second-order Fourier regression $\left(R_{e}\right)$ (black line) and derived as the intercept of the rectangular hyperbola fitted to the light response curve (Net Ecosystem Exchange (NEE) versus Photosyntetic Active Radiation (PAR) without u* threshold correction ( $\left.R_{\text {eluE }}\right)$ (blue line). (b) Gross Ecosystem Productivity, GEP (gC m-2 d-1) derived using $R_{e}$ (black line); and using $R_{e l u E}, G E P_{L U E}(\mathrm{blue} \mathrm{line).}$ Grey boxes indicate Southern Hemisphere spring and summer October to April. 


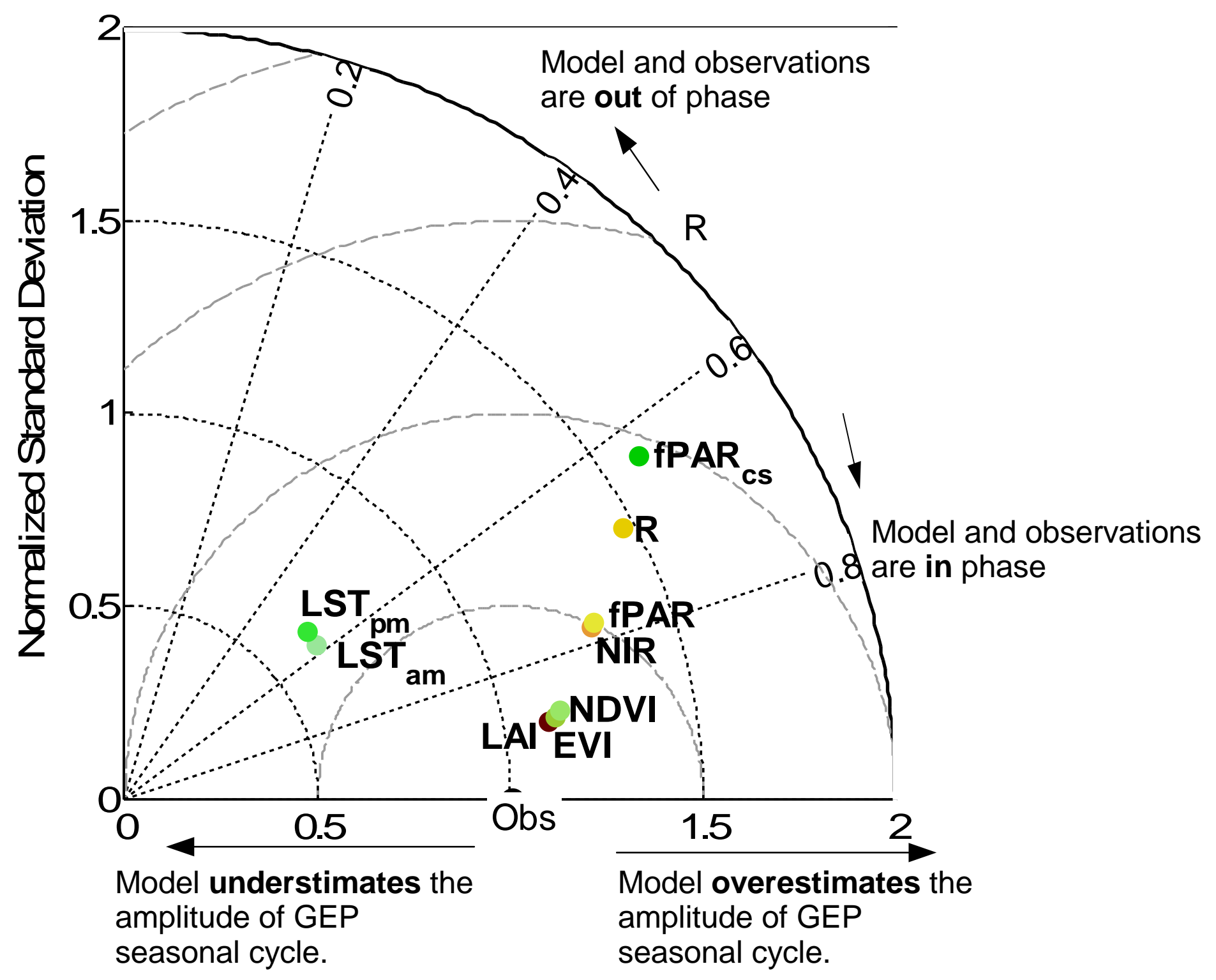

Supplement Figure 2. Taylor diagram comparing Howard Springs eddy flux tower measured Gross Ecosystem Productivity (GEP) and GEP based on MODIS and AVHHR products (based on a Type II linear regression). Labels indicate MODIS Leaf Area Index (LAI) and PPAR (fPAR); MODIS NBAR (nadir) Normalized Vegetation Index (NDVI43), Enhanced Vegetation Index (EVI43), Green reflectance (G43), Red (R43), Near Infrared (NIR43); MODIS Land Surface Temperature Daytime (LSTam) and Nighttime (LSTpm) and AVHHR (processed by CSIRO) total fPAR (fPARcs). 
HSP: Savanna
2000-2011
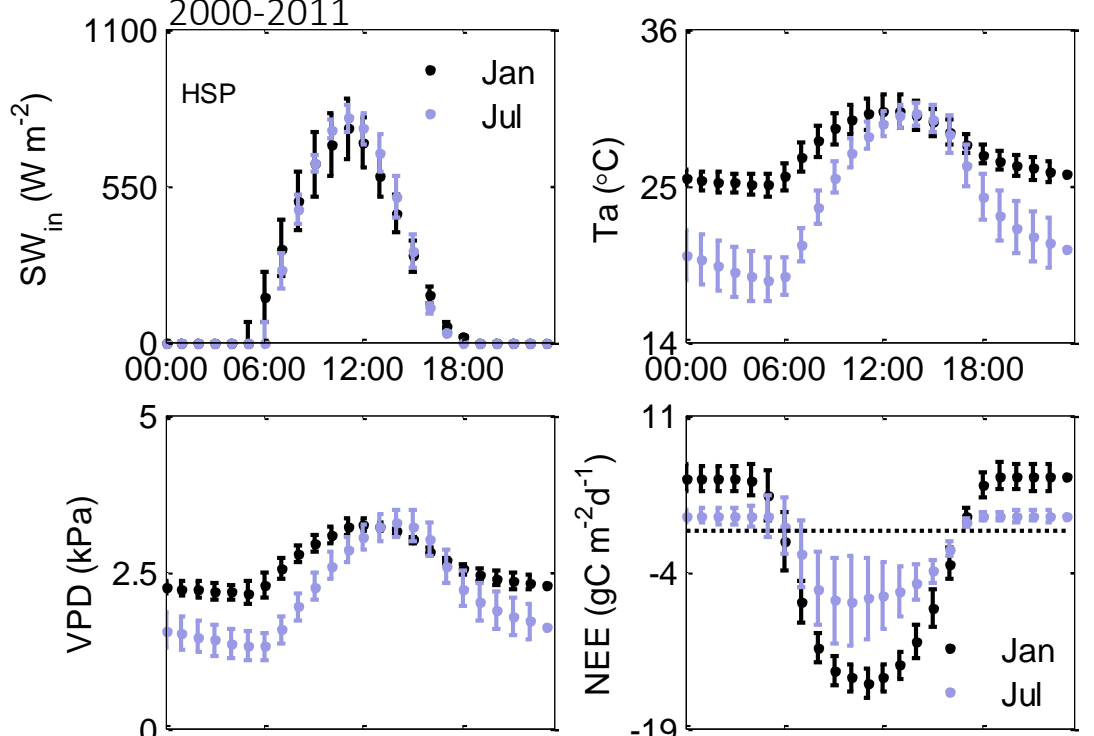

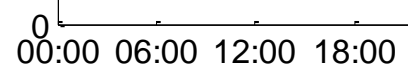

ASP: Mulga woodland

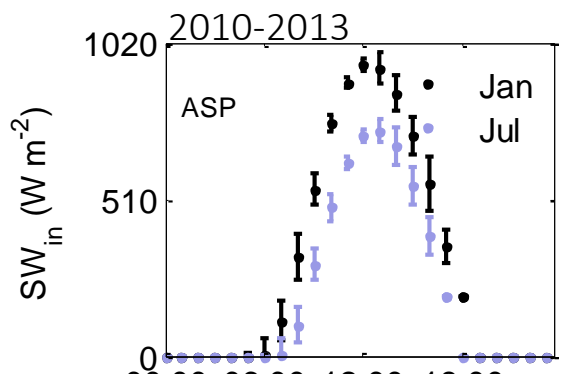

00:00 06:00 12:00 18:00

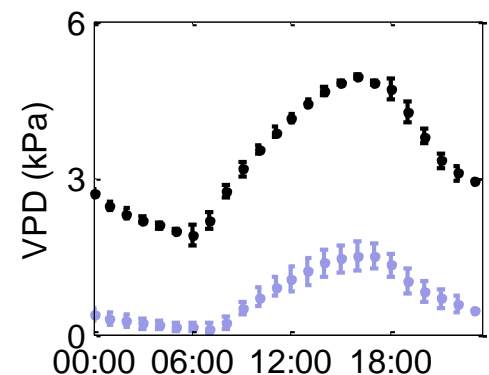

14:00 06:00 12:00 18:00
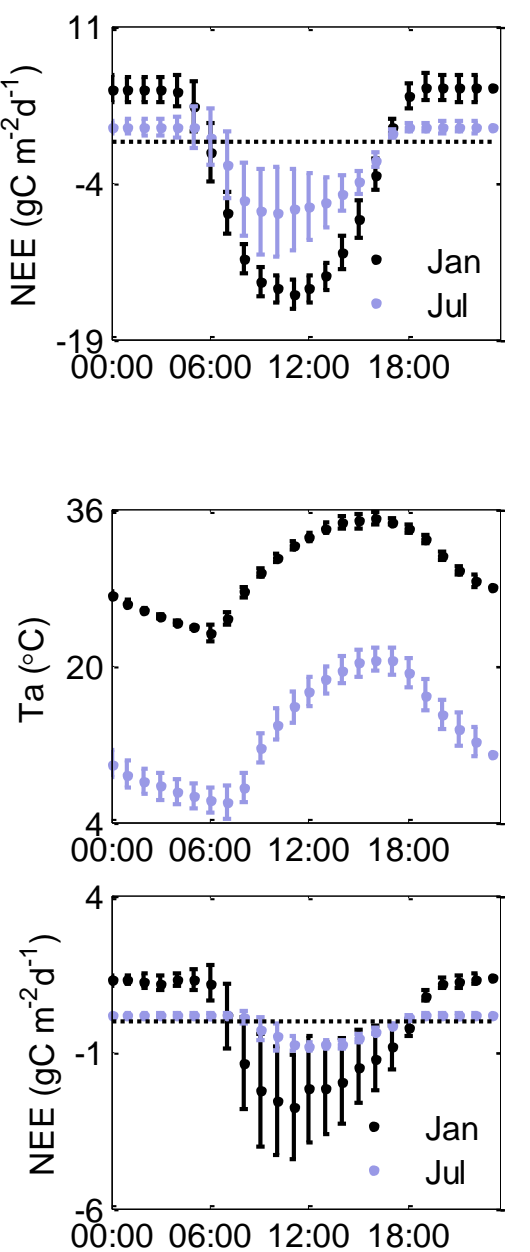
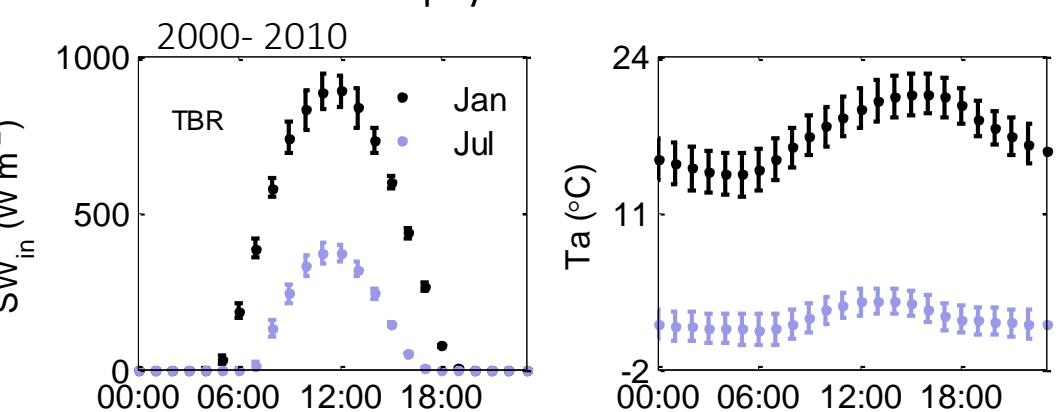

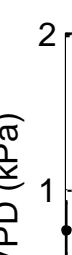

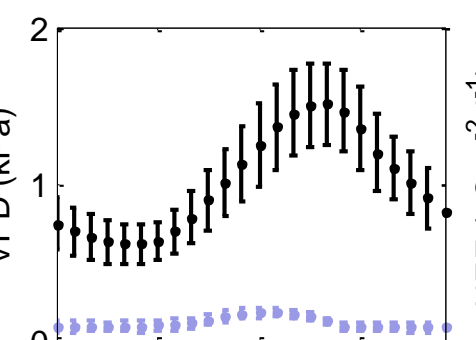

00:00 06:00 12:00 18:00

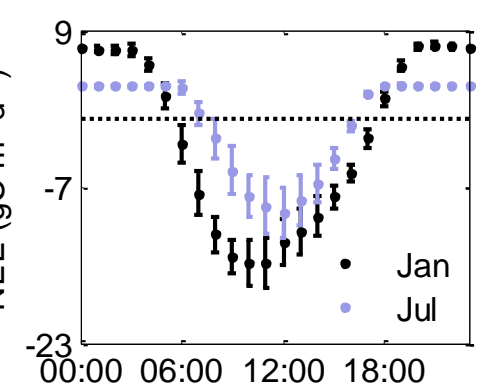

$\mathrm{CHO}$ : Mediterranean - Mallee
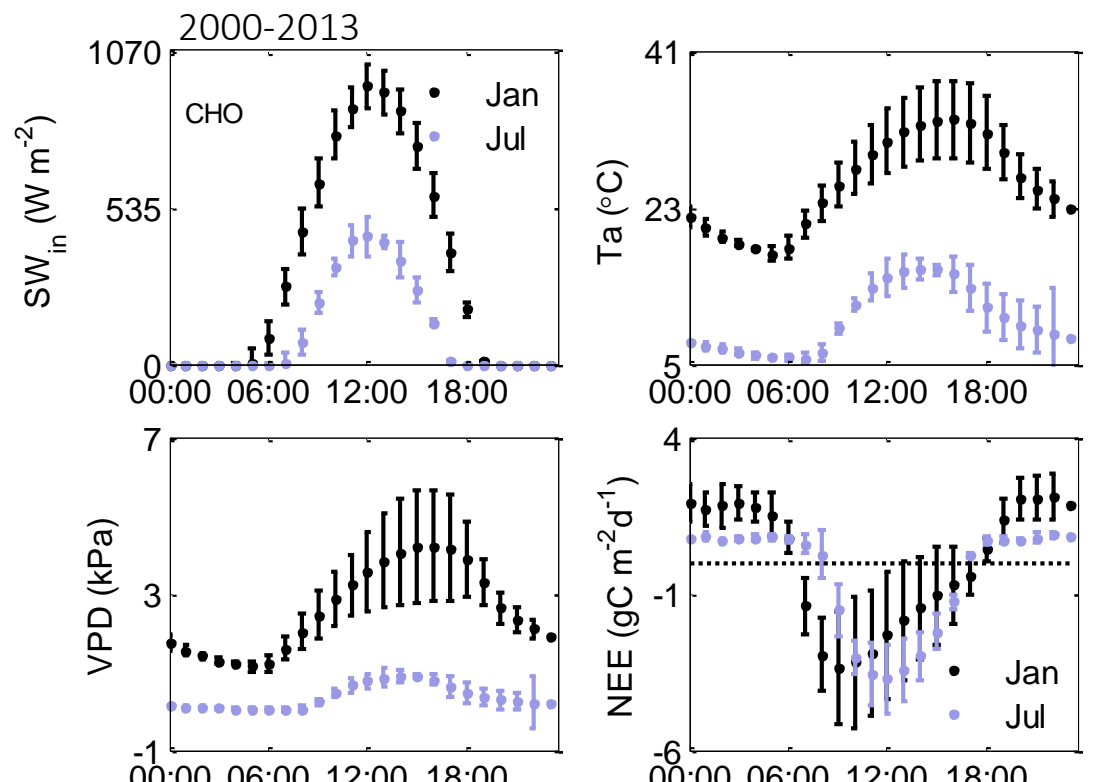

Supplement Figure 3. Diel cycle January (Australian winter) and July (summer) red dot mean and error bars indicating standard deviation for all January or July months (missing if only one year of data is available). Four plots per site from right to left ,top to bottom, Photosyntetic Active Radiation, PAR (umol m-2 s-1); Gross Ecosystem Productivity, GEP (gC m-2 d-1); GEP/PAR (gC/MJ) and Net Ecosystem Exchange, NEE (gC m-2 d-1). HSP: Howard Springs Savanna, TBR: Tumbarumba wet schlerophill forest, ASP, Alice Springs Mulga and CHO, Calperum-Chowilla. 

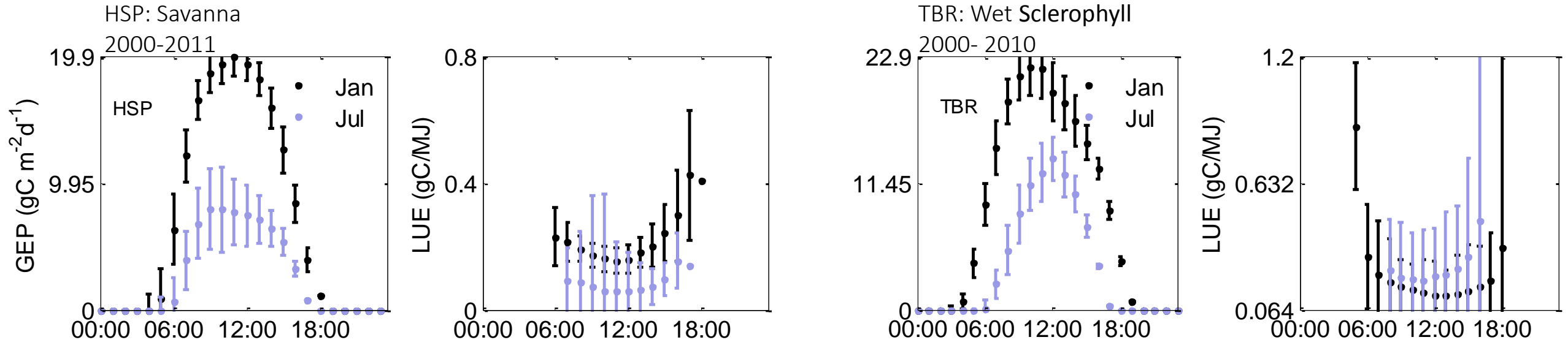

ASP: Mulga woodland
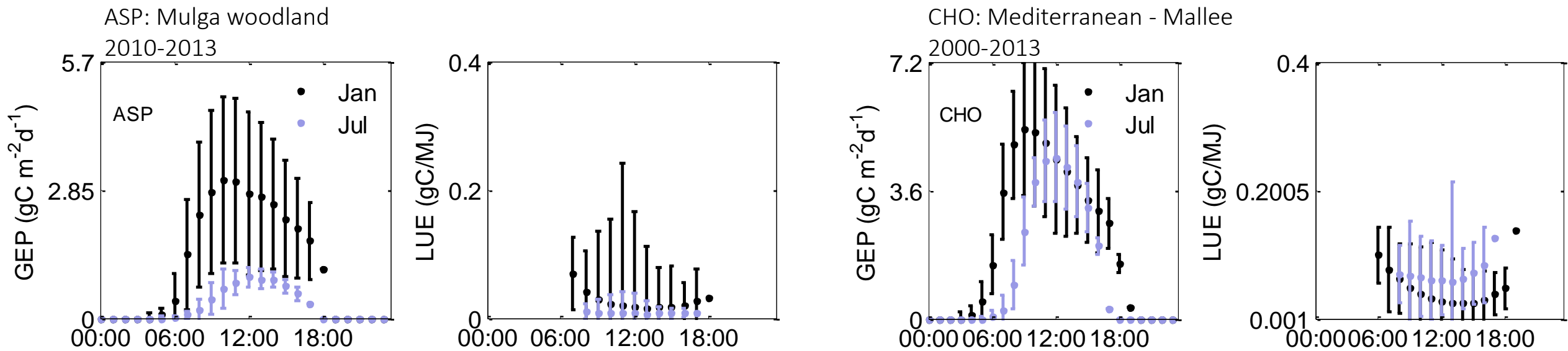

Supplement Figure 4. Diel cycle January (Australian winter) and July (summer) red dot mean and error bars indicating standard deviation for all January or July months (missing if only one year of data is available). Four plots per site from right to left ,top to bottom, Photosyntetic Active Radiation, PAR (umol m-2 s-1); Gross Ecosystem Productivity, GEP (gC m-2 d-1); GEP/PAR (gC/MJ) and Net Ecosystem Exchange, NEE (gC m-2 d-1). HSP: Howard Springs Savanna, TBR: Tumbarumba wet schlerophill forest, ASP, Alice Springs Mulga and CHO, Calperum-Chowilla. 

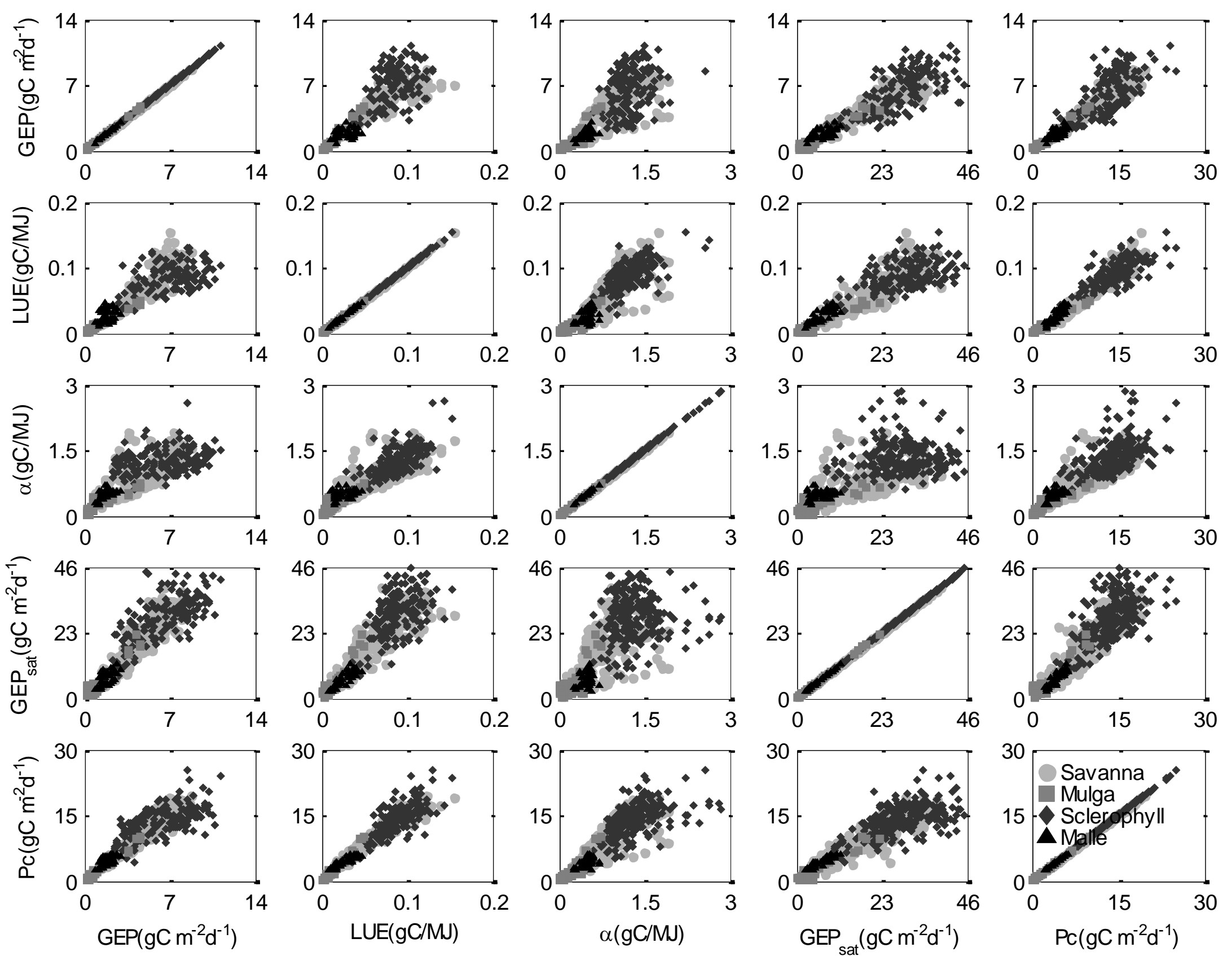

Supplement Figure 5. Multi-site regressions between different measures of ecosystem function, where GEP is Gross Ecosystem Productivity, GEP/PAR is the ratio of GEP to incoming Photosynthetic Active Radiation, LUE is Light Use Efficiency, GEP sat is GEP at saturation light and $P C$ is the photosynthetic capacity. 

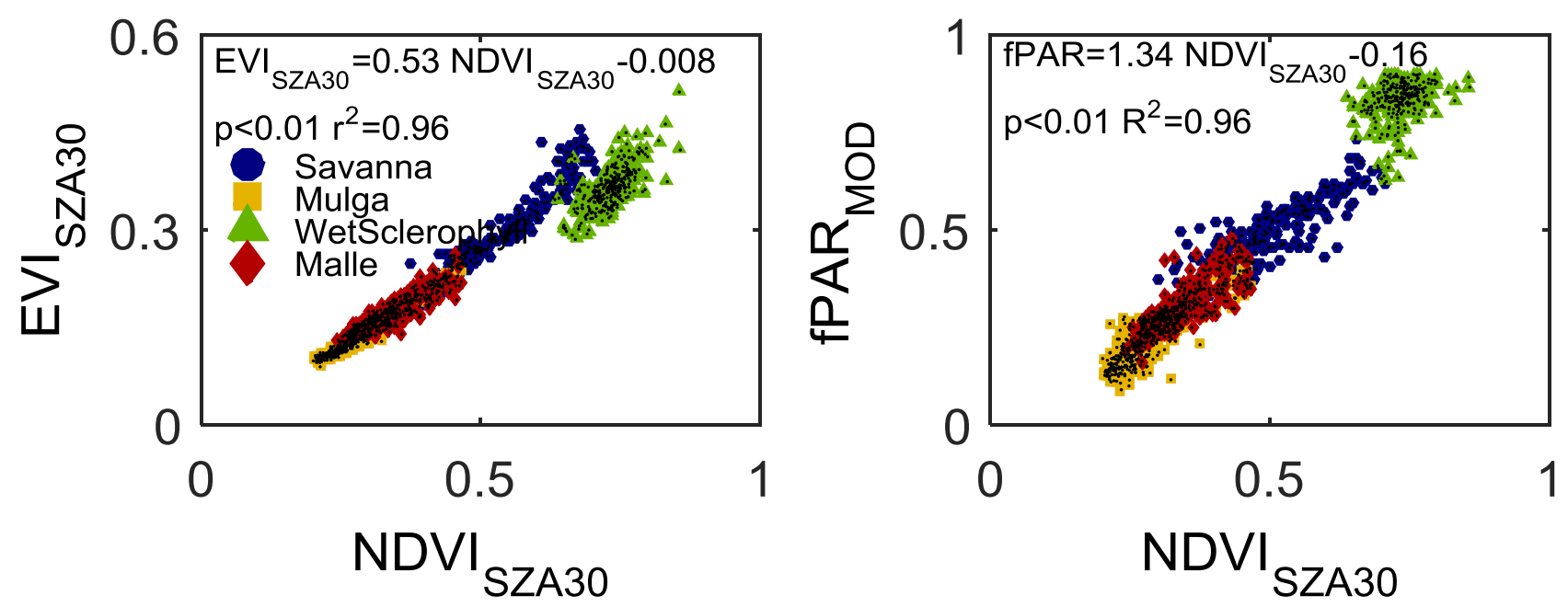

Supplement Figure 7. Multi-site regressions between MODIS Normalized Difference Vegetation Index, NDVI $I_{\text {SZAзO }}$ at fixed solar zenith angle of $30^{\circ}$ and Enhanced Vegetation Index, EVI $I_{\text {SZA30 }}$ (left panel) and MODIS fraction of the absorbed Photosynthetic Active Radiation, fPAR (right panel) 

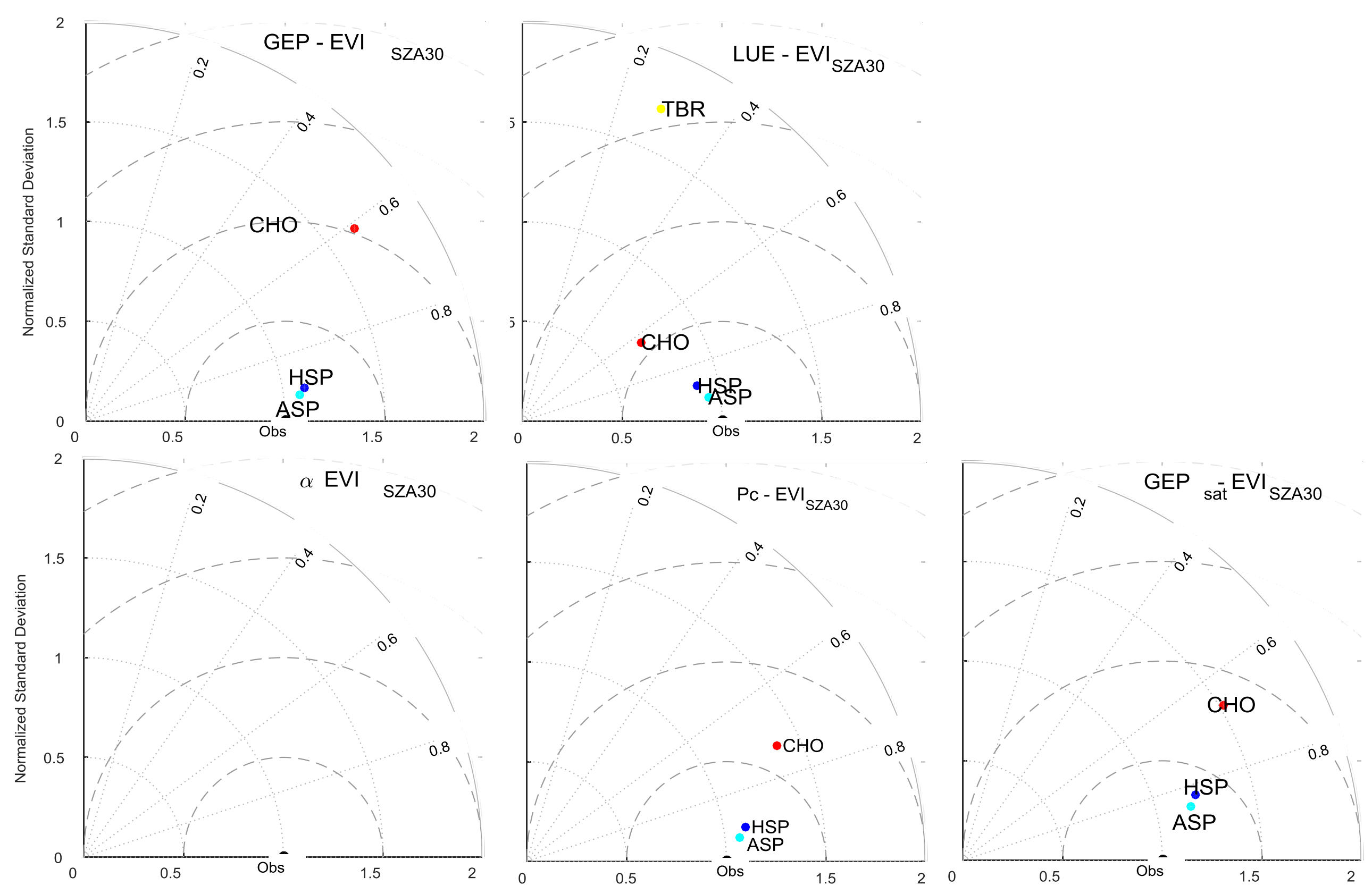

Supplement Figure 7. Taylor diagrams showing linear moc

(HSP), Tumbarumba (TMB), Alice Springs (ASP) and Calperum-Chowilla (CHO) based on sitespecific linear regressions between Gross Ecosystem Productivity (GEP), Light Use Efficiency (LUE), Photosynthetic Capacity (Pc), ecosystem quantum yield ( $\alpha$ ) and daytime Net Ecosystem Exchange $\left(N E E_{d a y}\right)$ and MODIS Enhanced Vegetation Index at fixed solar zenith angle of $30^{\circ}\left(E_{\mathrm{VI}} \mathrm{IZAO}_{\mathrm{O} O}\right)$. Missing sites indicate that the model overestimates the seasonality of observations -model normalized standard deviation is $>2$. 


\begin{tabular}{|l|l|l|}
\hline SDS Name & Field & Value Masked \\
\hline Pixel Reliability & N/A & -1 (Fill/No Data) \\
\hline & & 2 (Snow/lce) \\
\hline VI Quality & 3 (Cloudy) \\
\hline & MODLAND_QA & 10 (Produced/Cloudy) \\
\hline & & 11 (Not Produced) \\
\hline & VIUsefulness & 1100 (Lowest Quality) \\
\hline & & 1101 (Not Useful) \\
\hline & & 1110 (Data Faulty) \\
\hline & & 1111 (Other) \\
\hline & & \\
\hline
\end{tabular}




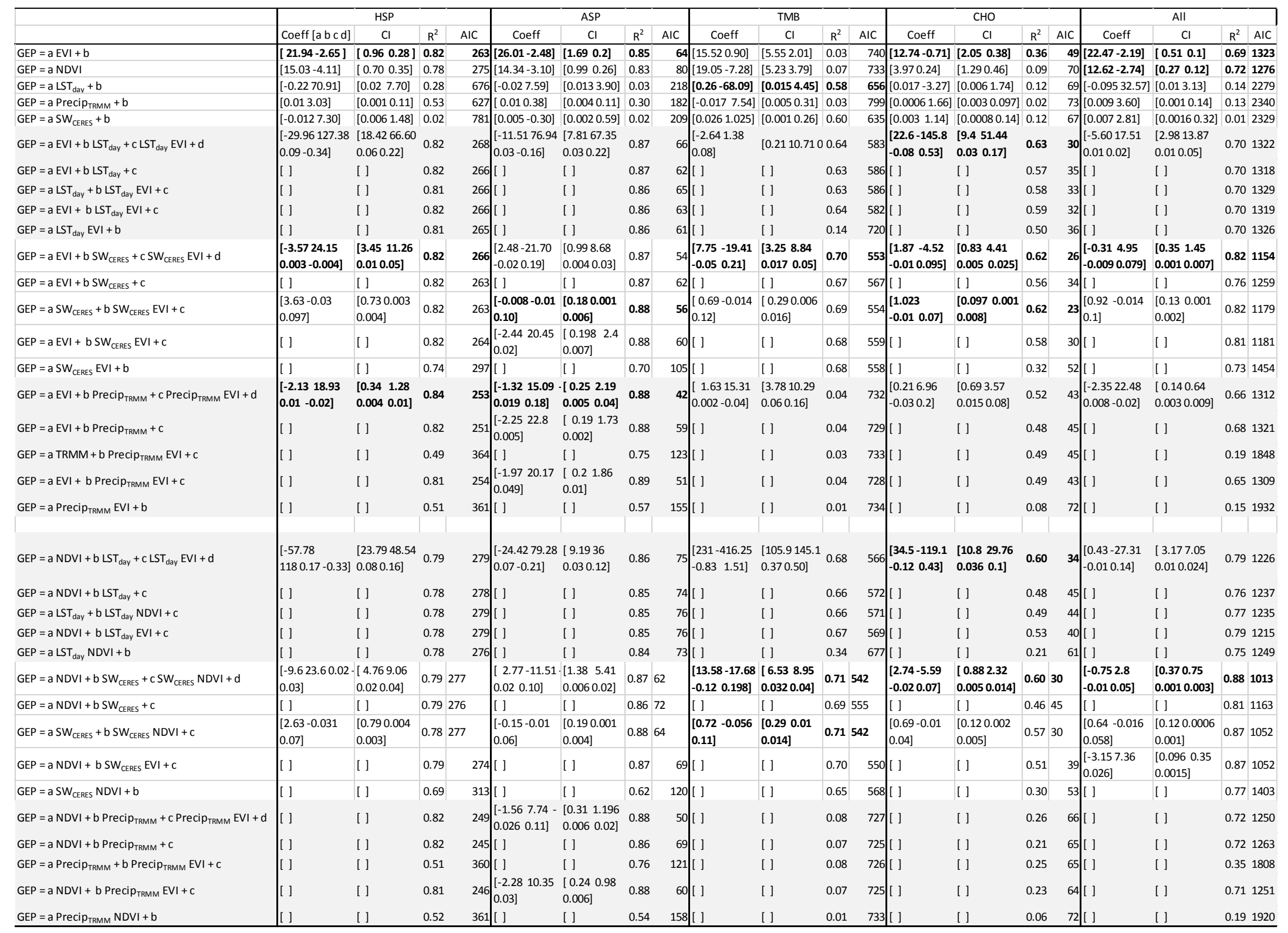

Supplement Table 3. Linear regressions obtained by a nonlinear mixed-effects regression model for Gross Ecosystem Productivity (GEP) versus combinations of 16-day average MODIS products: fixed solar zenith angle of $30^{\circ}$ Enhanced Vegetation Index (EVI), daytime and Land Surface Temperature (LST day), fixed solar zenith angle of $30^{\circ}$ Normalized Difference Vegetation Index (NDVI), precipitation from the Tropical Rainfall Measuring Mission (TRMM) data product from 1998-2013 (NASA, 2014) (mm mo-1), and surface shortwave incident radiation from the CERES (Kato et al., 2012). Model runs for HSP: Howard Springs, ASP: Alice Springs Mulga, CHO: Calperum-Chowilla, and TBR: Tumbarumba and all available data (includes all sites). EVI and NDVI labels are used instead of $\mathrm{EVI}_{\mathrm{SZA} 30}$ and $\mathrm{NDVI}_{\mathrm{SZA} 30}$ for displaying purposes. 


\begin{tabular}{|c|c|c|c|c|c|c|c|c|c|c|c|c|c|c|c|c|c|c|c|c|}
\hline & HSP & & & & ASP & & & & TMB & & & & $\mathrm{CHO}$ & & & & All & & & \\
\hline & Coeff [a b] & $\mathrm{Cl}$ & $\mathrm{R}^{2}$ & RMSE & Coeff [a b] & $\mathrm{Cl}$ & $\mathrm{R}^{2}$ & RMSE & Coeff [a b] & $\mathrm{Cl}$ & $\mathrm{R}^{2}$ & RMSE & Coeff $[a b]$ & $\mathrm{Cl}$ & $\mathrm{R}^{2}$ & RMSE & Coeff [a b] & $\mathrm{Cl}$ & $\mathrm{R}^{2}$ & RMSE \\
\hline $\mathrm{LUE}=\mathrm{aEVI} \mathrm{SZA30}_{\mathrm{SA}}+\mathrm{b}$ & 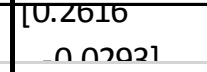 & {$[0.0124 \quad 0.00$} & 0.77 & 70.0006 & $5[0.3-0.029]$ & {$[0.020 .002]$} & 0.85 & 0.000 & {$[\mathrm{l}$} & [ ] & 0.10 & 0.000 & {$[1]$} & [ ] & 0.44 & 40.0000 & {$[1]$} & [ ] & 0.67 & 0.000 \\
\hline$L U E=a N D V I_{S Z A 30}+b$ & [ ] & [ ] & 0.73 & 30.0000 & {$[$ ] } & [ ] & 0.83 & 0.000 & {$[$ ] } & [ ] & 0.13 & 0.000 & {$[0.18-0.041]$} & {$[0.0160 .006]$} & 0.69 & 90.0000 & {$[$ ] } & [ ] & 0.75 & 0.000 \\
\hline$L U E=a L A I+b$ & [ ] & [ ] & 0.75 & 50.0000 & {$[$ ] } & [ ] & 0.68 & 0.000 & {$[$ ] } & [ ] & 0.12 & 0.000 & {$[$ ] } & {$[$ ] } & 0.52 & 20.0005 & {$[$ ] } & [ ] & 0.73 & 0.004 \\
\hline$L U E=a f P A R+b$ & [ ] & [ ] & 0.74 & 40.0001 & {$[$ ] } & [ ] & 0.65 & 0.000 & {$[$ ] } & [ ] & 0.15 & 0.001 & {$[0.13-0.02]$} & [0.007 0.002] & 0.85 & 50.0000 & {$[$ ] } & [ ] & 0.78 & 0.000 \\
\hline$P c=a E V I_{S Z A 30}+b$ & {$[50.5671$} & {$\left[\begin{array}{ll}2.1125 & 0.61\end{array}\right.$} & 10.81 & 0.0912 & {$[60.91-5.91]$} & [3.42 0.41] & 0.87 & 0.000 & {$[44.26-2.34]$} & [7.12 2.6] & 0.15 & 3.314 & {$[$ ] } & [ ] & 0.52 & 20.0340 & {$[49.43-4.75]$} & [0.82 0.18] & 0.81 & 0.000 \\
\hline$P c=a N D V I_{S Z A 30}+b$ & [ ] & [ ] & 0.76 & 0.0000 & {$[33.78-7.40]$} & {$\left[\begin{array}{lll}1.95 & 0.51]\end{array}\right.$} & 0.85 & 0.000 & {$[42.27-17.05]$} & [6.72 4.9] & 0.16 & 0.145 & {$[$ ] } & [ ] & 0.49 & 90.0000 & {$[27.47-5.81]$} & [0.43 0.19] & 0.79 & 0.000 \\
\hline$P c=a L A I+b$ & [ $7.66-2.57]$ & {$\left[\begin{array}{lll}0.28 & 0.38\end{array}\right]$} & 0.79 & 0.0000 & {$[$ ] } & [ ] & 0.73 & 0.000 & {$[\mathrm{l}$} & [ ] & 0.10 & 0.000 & {$[$ ] } & [ ] & 0.68 & 80.0000 & {$[$ ] } & [ ] & 0.64 & 0.432 \\
\hline$P c=a f P A R+b$ & [ ] & [ ] & 0.72 & 0.0000 & {$[$ ] } & [ ] & 0.65 & 0.000 & {$[$ ] } & [ ] & 0.03 & 0.000 & {$[$ ] } & [ ] & 0.66 & 60.0000 & {$[$ ] } & [ ] & 0.74 & 0.000 \\
\hline$\alpha=a E V I_{S Z A 30}+b$ & [ ] & [ ] & 0.34 & 0.0035 & {$[$ ] } & [ ] & 0.75 & 0.000 & {$[$ ] } & [ ] & 0.01 & 0.000 & {$[$ ] } & [ ] & 0.18 & 80.0055 & {$[1]$} & [ ] & 0.54 & 0.000 \\
\hline$\alpha=a N^{\prime} V I_{S Z A 30}+b$ & [ ] & [ ] & 0.29 & 0.0000 & {$[$ ] } & [ ] & 0.74 & 0.000 & {$[$ ] } & [ ] & 0.02 & 0.000 & {$[$ ] } & [ ] & 0.09 & 90.0064 & {$[$ ] ] } & [ ] & 0.58 & 0.000 \\
\hline$\alpha=a L A I+b$ & [ ] & [ ] & 0.32 & 0.0014 & {$[$ ] } & [ ] & 0.72 & 0.000 & {$[$ ] } & [ ] & 0.02 & 0.000 & {$[$ ] } & [ ] & 0.26 & 50.0000 & {$[$ ] } & [ ] & 0.54 & 0.057 \\
\hline$\alpha=a f P A R+b$ & [ ] & [ ] & 0.28 & 30.0000 & {$[$ ] } & [ ] & 0.55 & 0.000 & {$[$ ] } & [ ] & 0.02 & 0.000 & {$[$ ] } & [ ] & 0.10 & 0.0000 & {$[$ ] } & [ ] & 0.62 & 0.006 \\
\hline $\mathrm{GEP}_{\mathrm{sat}}=\mathrm{aEVI} \mathrm{IZA} 30+\mathrm{b}$ & [ ] & [ ] & 0.66 & 0.3131 & {$[$ ] ] } & [ ] & 0.73 & 0.000 & {$[113.95-12.36]$} & [17.96.5] & 0.14 & 0.132 & {$[$ ] } & [ ] & 0.44 & 40.1384 & {$[\mathrm{l}]$} & [ ] & 0.71 & 0.000 \\
\hline $\mathrm{GEP}_{\text {sat }}=\mathrm{a} \mathrm{NDVI}_{\mathrm{SZA30}}+\mathrm{b}$ & {$[$ ] } & [ ] & 0.69 & 0.2707 & {$[$ ] } & [ ] & 0.71 & 0.000 & {$[$ ] } & [ ] & 0.13 & 0.362 & {$[$ ] } & [ ] & 0.51 & 10.0000 & {$[$ ] } & [ ] & 0.74 & 0.000 \\
\hline GEPsat $=a \mathrm{LAI}+\mathrm{b}$ & [ ] ] & [ ] & 0.69 & 0.3214 & {$[$ ] } & [ ] & 0.58 & 0.000 & {$[$ ] } & [ ] & 0.07 & 0.356 & {$[$ ] } & [ ] & 0.60 & 0.1019 & {$[$ ] } & [ ] & 0.63 & 1.539 \\
\hline GEPsat $=a$ fPAR $+b$ & [ ] & [ ] & 0.65 & 0.2730 & {$[$ ] } & [ ] & 0.55 & 0.000 & {$[$ ] } & [ ] & 0.01 & 0.497 & [ ] & [ ] & 0.72 & 20.0000 & {$[$ ] } & [ ] & 0.68 & 0.162 \\
\hline$G E P=a E V I_{\text {SZA30 }}+b$ & [23.03-2.89] & {$[0.960 .28]$} & 0.82 & 0.0502 & {$[26.17-2.49]$} & {$\left[\begin{array}{ll}1.69 & 0.2\end{array}\right]$} & 0.86 & 0.000 & {$[$ ] } & [ ] & 0.04 & 0.129 & [ ] & [ ] & 0.37 & 70.0000 & {$[$ ] } & [ ] & 0.68 & 0.000 \\
\hline$G E P=a N D V I_{S Z A 30}+b$ & {$[16.27-4.63]$} & {$\left[\begin{array}{lll}0.70 & 0.35\end{array}\right]$} & 0.77 & 0.0000 & [14.33 -3.09] & {$\left[\begin{array}{ll}0.99 & 0.26\end{array}\right]$} & 0.83 & 0.000 & {$[$ ] } & [ ] & 0.06 & 0.000 & [ ] & [ ] & 0.10 & 0.0000 & {$[$ ] } & [ ] & 0.70 & 0.000 \\
\hline GEP $=a L A I+b$ & [ ] & [ ] & 0.84 & 0.0406 & {$[$ ] } & [ ] & 0.74 & 0.000 & {$[$ ] } & [ ] & 0.04 & 0.090 & [ ] & [ ] & 0.37 & 70.0000 & {$[$ ] } & [ ] & 0.60 & 0.395 \\
\hline GEP $=a$ fPAR $+b$ & [ ] & [ ] & 0.71 & 0.0230 & {$[$ ] } & [ ] & 0.63 & 0.000 & {$[$ ] } & [ ] & 0.00 & 0.000 & [ ] & [ ] & 0.07 & 70.0337 & [ ] & [ ] & 0.65 & 0.000 \\
\hline
\end{tabular}

RMSE: The square root of the estimated error variance (computed on the log scale for the exponential error model)

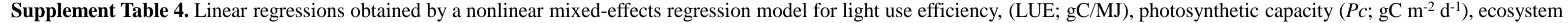

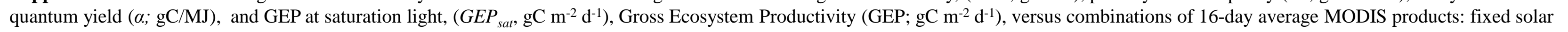

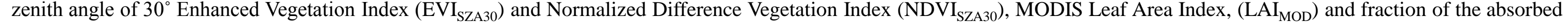
Photosynthetic Active Radiation ( fPAR $_{\mathrm{MOD}}$ ) 